\title{
Massively parallel approximate Gaussian process regression
}

\author{
Robert B. Gramacy \\ Booth School of Business \\ The University of Chicago \\ rbgramacy@chicagobooth.edu \\ Jarad Niemi \\ Department of Statistics \\ Iowa State University \\ niemi@iastate.edu \\ Robin M. Weiss \\ Research Computing Center \\ The University of Chicago \\ robinweiss@uchicago.edu
}

\begin{abstract}
We explore how the big-three computing paradigms - symmetric multi-processor (SMP), graphical processing units (GPUs), and cluster computing - can together be brought to bear on large-data Gaussian processes (GP) regression problems via a careful implementation of a newly developed local approximation scheme. Our methodological contribution focuses primarily on GPU computation, as this requires the most care and also provides the largest performance boost. However, in our empirical work we study the relative merits of all three paradigms to determine how best to combine them. The paper concludes with two case studies. One is a real data fluid-dynamics computer experiment which benefits from the local nature of our approximation; the second is a synthetic example designed to find the largest data set for which (accurate) GP emulation can be performed on a commensurate predictive set in under an hour.
\end{abstract}

Key words: emulator, nonparametric regression, graphical processing unit, symmetric multi-processor, cluster computing, big data, computer experiment

\section{Introduction}

Gaussian processes (see, e.g., Rasmussen and Williams, 2006) form the canonical regression model for data arising from computer experiments (Santner et al., 2003). Their nonparametric flexibility, interpolative capability, and conditionally analytic predictive distributions with high accuracy and appropriate coverage make them ideally suited to applications where accuracy, and full uncertainty quantification/propagation, are equally important. Some examples include design (Santner et al., 2003), sequential design (Seo et al., 2000), optimization (Jones 
et al., 1998), contour finding (Ranjan et al., 2011), and calibration (Kennedy and O'Hagan, 2001; Bayarri et al., 2007), to name just a few.

The main disadvantage to Gaussian process (GP) regression models is computational. Inference and prediction require decomposing an $N \times N$ matrix, for $N$ observations, and that usually requires an $O\left(N^{3}\right)$ operation. With modern desktop computers, that limits GPs to $N$ in the low thousands for point inference (e.g., via maximum likelihood estimation [MLE] or cross validation $[\mathrm{CV}]$ ), and to the hundreds for sampling methods (Bayesian Monte Carlo or bootstrap). Point inference can be pushed to $N$ in the tens of thousands when modern supercomputer computing facilities are paired with new distributed linear algebra libraries, as illustrated by Paciorek et al. (2013).

As data sets become ever larger, research into approximate GP modeling has become ever more frenzied. Early examples include the works of Vecchia (1988); Nychka et al. (2002); Stein et al. (2004); Quiñonero-Candela and Rasmussen (2005); Furrer et al. (2006); Cressie and Johannesson (2008). More recent examples include those of Haaland and Qian (2011); Sang and Huang (2012); Kaufman et al. (2012); Eidsvik et al. (2013). Sparsity is a recurring theme. The approximations involve either explicitly working with a subset of the data, or imposing a covariance structure which produces sparse matrices that can be quickly decomposed. Another way to get fast inference is to impose structure on the design, e.g., forcing a lattice design. This can lead to substantial shortcuts in the calculations (e.g., Gilboa et al., 2012; Plumlee, 2013), but somewhat limits applicability.

In this work we consider the particular approximation suggested by Gramacy and Apley (2014). That approximation drew inspiration from several of the works referenced above, but primarily involves modernizing an old idea of local kriging neighborhoods (Cressie, 1991 , pp. 131-134) by borrowing from active learning heuristics for sequential design (Seo et al., 2000). The idea is to build a GP predictor for a particular location, $x$, by greedily building a sub-design $X_{n}(x) \subseteq X$, where $n \ll N$, according to an appropriate criteria. Then, prediction over a vast grid can be parallelized by processing each element, $x$, of the grid independently of the others. Such independence can also yield a thrifty nonstationary modeling feature.

Our primary contribution centers around recognizing that the criteria, which must be repeatedly evaluated over (nearly) the entire design space $X$, can be implemented on a graphical processing unit (GPU). In essence, we are proposing to nest a parallel (GPU) computation within an already parallelized symmetric multiprocessor (SMP) environment. Both the GPU implementation (in CUDA), and its interface to the outer parallel scheme (via OpenMP), must be treated delicately to be efficient. We then round out the supercomputing trifecta by distributing computation on a cluster of multi-core, and multi-GPU, nodes.

GPUs offer great promise in scientific computing, in some cases leading to 100x speedups. We find more modest speedups in our examples (40-60x), echoing similar results obtained with GPU-accellerated large matrix operations (Franey et al., 2012, Eidsvik et al., 2013, Paciorek et al., 2013). In contrast to these works, however, we do not make use of library routines. In fact, our approximations explicitly keep the required matrices small. Our repetitive local searches involve low-level operations which can be implemented on the GPU with a very small (and completely open source) kernel. The entire implementation, including 
CUDA, C, and R subroutines, is made available as an $\mathrm{R}$ package called laGP (Gramacy, 2013).

The remainder of the paper is outlined as follows. Section 2 reviews GP computer modeling generally, and the Gramacy and Apley (2014) local approximate GP scheme specifically, with focus on the particular subroutine that is re-implemented in this paper. Section 3 details our CUDA implementation of that subroutine, and in Section 4 we study the speedups obtained in isolation (i.e., compared to a CPU version of the same subroutine), and within the wider context (incorporating the calling environment on a multi-core, multi-GPU compute node) on a toy problem. In Section 5 we augment with an off-the-shelf, simple network of workstations (SNOW), cluster computing facility in order to emulate a large real-data computer experiment from the literature. We then separately consider a synthetic datagenerating mechanism to find the largest problem we can solve with an hour of (multi-node cluster) computing time. The paper concludes with a brief discussion in Section 6 .

\section{Computer model emulation}

Computer simulation of a system under varying conditions represents a comparatively inexpensive alternative to actual physical experimentation and/or monitoring. Examples include aeronautics (designing a new aircraft wing) and climate science (collecting atmospheric ozone data). In some cases it is the only (ethical) alternative, e.g., in epidemiology. Still, computer models can be complex and computationally demanding, and therefore only a limited (if still vast) number of runs $D_{N}=\left(x_{1}, y_{1}\right), \ldots,\left(x_{N}, y_{N}\right)$ for input conditions $x_{i}$, producing outputs $y_{i}$, can be obtained. Computer model emulation, therefore, remains an integral component of many applications involving data arising from computer simulation. Given the data $D_{N}$, an emulator provides a distribution over possible responses $Y(x) \mid D_{N}$ for new inputs $x$. A key requirement is that the emulator be able to provide that distribution at much lower computational expense than running new simulations.

\subsection{Gaussian process regression}

The GP regression model is canonical for emulation, primarily for the following two reasons.

1. The predictive equations $p\left(y(x) \mid D_{N}, K_{\theta}\right)$ have a closed form given a small number of "tuning" parameters, $\theta$, describing the correlation structure $K_{\theta}(\cdot, \cdot)$, which is discussed separately below. They are Student- $t$ with degrees of freedom $N$,

$$
\begin{aligned}
& \text { mean } \quad \mu\left(x \mid D_{N}, \theta\right)=k^{\top}(x) K^{-1} Y, \\
& \text { and scale } \quad \sigma^{2}\left(x \mid D_{N}, \theta\right)=\frac{\psi\left[K(x, x)-k^{\top}(x) K^{-1} k(x)\right]}{N} \text {, }
\end{aligned}
$$

where $k^{\top}(x)$ is the $N$-vector whose $i^{\text {th }}$ component is $K_{\theta}\left(x, x_{i}\right), K$ is an $N \times N$ matrix whose entries are $K_{\theta}\left(x_{i}, x_{j}\right)$, and $\psi=Y^{\top} K^{-1} Y$. Using properties of the Student- $t$, the variance of $Y(x)$ is $V(x) \equiv \mathbb{V} \operatorname{ar}\left[Y(x) \mid D_{N}, \theta\right]=\sigma^{2}\left(x \mid D_{N}, \theta\right) \times N /(N-2)$. 
Observe that the mean is a linear predictor, which depends on the responses $Y$, and that the variance is independent of $Y$ given $K_{\theta}(\cdot, \cdot)$. The result is a "football-shaped" predictive interval which is wide away from data locations $x_{i}$, and narrows at the $x_{i}-\mathrm{a}$ visually appealing feature for an emulator.

2. Maximum likelihood inference for $\theta$ is straightforward given analytic forms of the (marginalized) likelihood equations

$$
p(Y \mid \theta)=\frac{\Gamma[N / 2]}{(2 \pi)^{N / 2}|K|^{1 / 2}} \times\left(\frac{\psi}{2}\right)^{-\frac{N}{2}},
$$

whose derivatives, for Newton-like optimization, are also available analytically.

Together, analytic prediction and straightforward optimization for inference, make for a relatively easy implementation of a non-parametric regression.

The choice of correlation structure, $K_{\theta}(\cdot, \cdot)$, can have a substantial impact on the nature of inference and prediction, restricting the smoothness of the functions and controlling a myriad of other aspects. However there are several simple default choices that are popular in the literature. In this paper we use an isotropic Gaussian correlation $K_{\theta, \eta}\left(x, x^{\prime}\right)=$ $\exp \left\{-\left\|x-x^{\prime}\right\|^{2} / \theta\right\}$, where $\theta$ is called the lengthscale parameter. We make this choice purely for simplicity of the exposition, and because it is historically the most common choice for computer experiments. The general methodology we present is independent of this choice.

Unfortunately, the above equations reveal a computational expense that depends on the size of the correlation matrix, $K$. Inverse and determinant calculations are $O\left(N^{3}\right)$ which, even for modest $N$, can mean that (in spite of the above attractive features) GPs may not satisfy the key requirement of being fast relative to the computer simulation being emulated. Advances in hardware design, e.g., multi-core machines and GPUs, may offer some salvation. Recently several authors (Franey et al., 2012; Eidsvik et al., 2013; Paciorek et al., 2013) have described custom GP prediction and inference schemes which show a potential to handle much larger problems than ever before.

\subsection{Local approximate Gaussian process modeling}

It makes sense to develop emulators which can exploit these new resources, especially as they move into the mainstream. For obvious reasons, emulation in better than $O\left(N^{3}\right)$ time is also desirable, and for that imposing sparsity is a popular tactic. Gramacy and Apley (2014) proposed a local scheme leveraging sparsity towards providing fast and accurate prediction, ideal for computer model emulation on modern multi-core desktops.

The idea is to focus, specifically, on the prediction problem at a particular location, $x$. Gramacy and Apley recognized, as many others have before, that data with inputs far from $x$ have vanishingly small influence on the resulting GP predictor (assuming typical distancebased correlation functions). Exploiting that, the scheme seeks to build a GP predictor from data $D_{n}(x) \equiv D_{n}\left(X_{n}(x)\right)$ obtained on a sub-design $X_{n}(x)$ of the full design $X \equiv X_{N}$, where $n \ll N$. One option is a so-called nearest neighbor $(\mathrm{NN})$ sub-design, where $D_{n}$ is comprised of 
the inputs in $X$ which are closest to $x$, measured relative to the chosen correlation function, but this is known to be sub-optimal (Vecchia, 1988). It is better to take at least a few design points farther away in order to obtain good estimates of the parameter $\theta$ (Stein et al., 2004). However, searching for the optimal design $\hat{D}_{n}(x)$, according to almost any criteria, is a combinatorially huge undertaking. The interesting pragmatic research question that remains is: is it possible to do better than the NN scheme without much extra computational effort?

Gramacy and Apley demonstrated that it is indeed possible, with the following greedy scheme. Suppose a local design $X_{j}(x), j<n$, has been built-up already, and that a GP predictor has been inferred from data $D_{j}(x)$. Then, choose $x_{j+1}$ by searching amongst the remaining unchosen design candidates $X_{N} \backslash X_{j}(x)$ according to a criterion, discussed momentarily. Augment the data set $D_{j+1}(x)=D_{j} \cup\left(x_{j+1}, y\left(x_{j+1}\right)\right)$ to include the chosen design point and its corresponding response, and update the GP predictor. Updating a GP predictor is possible in $O\left(j^{2}\right)$ time (Gramacy and Polson, 2011) with judicious application of the partitioned inverse equations (Barnett, 1979). So as long as each search for $x_{j+1}$ is fast, and involves no new operations larger than $O\left(j^{2}\right)$, then the final scheme, repeating for $j=n_{0}, \ldots, n$ will require $O\left(n^{3}\right)$ time, just like the NN scheme.

Gramacy and Apley considered two criteria in addition to NN, one being a special case of the other. The first is to minimize the empirical Bayes mean-square prediction error (MSPE): $J\left(x_{j+1}, x\right)=\mathbb{E}\left\{\left[Y(x)-\mu_{j+1}\left(x \mid D_{j+1}, \hat{\theta}_{j+1}\right)\right]^{2} \mid D_{j}(x)\right\}$ where $\hat{\theta}_{j+1}$ is the estimate for $\theta$ based on $D_{j+1}$. The predictive mean $\mu_{j+1}\left(x \mid D_{j+1}, \hat{\theta}_{j+1}\right)$ follows equation (1), except that the $j+1$ subscript has been added in order to indicate dependence on $x_{j+1}$ and the future, unknown $y_{j+1}$. They then derive the approximation

$$
J\left(x_{j+1}, x\right) \approx V_{j}\left(x \mid x_{j+1} ; \hat{\theta}_{j}\right)+\left(\left.\frac{\partial \mu_{j}(x ; \theta)}{\partial \theta}\right|_{\theta=\hat{\theta}_{j}}\right)^{2} / \mathcal{G}_{j+1}\left(\hat{\theta}_{j}\right) .
$$

The first term in (4) estimates predictive variance at $x$ after $x_{j+1}$ is added into the design,

$$
\begin{aligned}
V_{j}\left(x \mid x_{j+1} ; \theta\right) & =\frac{(j+1) \psi_{j}}{j(j-1)} v_{j+1}(x ; \theta), \\
\text { where } \quad v_{j+1}(x ; \theta) & =\left[K_{j+1}(x, x)-k_{j+1}^{\top}(x) K_{j+1}^{-1} k_{j+1}(x)\right] .
\end{aligned}
$$

Minimizing predictive variance at $x$ is a sensible goal. The second term in (4) estimates the rate of change of the predictive mean at $x$, weighted by the expected future inverse information, $\mathcal{G}_{j+1}\left(\hat{\theta}_{j}\right)$, after $x_{j+1}$ and the corresponding $y_{j+1}$ are added into the design. Note that this weight does not depend on $x$, but in weighting the rate of change (derivative) of the predictive mean at $x$ it is "commenting" on the value of $x_{j+1}$ for estimating the parameter of the correlation function, $\theta$. So this MSPE criteria balances reducing predictive variance with learning local wigglyness of the surface.

It turns out that the contribution of the second term, beyond the new reduced variance, is small. Gramacy and Apley show that the full MSPE criteria leads to qualitatively similar local designs $X_{n}(x)$ as ones obtained using just $V_{j}\left(x \mid x_{j+1} ; \hat{\theta}_{j}\right)$, which provides indistinguishable out-of-sample predictive performance at a fraction of the computational cost (since no 
derivative calculations are necessary). This simplified criteria is equivalent to choosing $x_{j+1}$ to maximize reduction in variance:

$$
\begin{aligned}
& v_{j}(x ; \theta)-v_{j+1}(x ; \theta) \\
& \quad=k_{j}^{\top}(x) G_{j}\left(x_{j+1}\right) m_{j}^{-1}\left(x_{j+1}\right) k_{j}(x)+2 k_{j}^{\top}(x) g_{j}\left(x_{j+1}\right) K\left(x_{j+1}, x\right)+K\left(x_{j+1}, x\right)^{2} m_{j}\left(x_{j+1}\right),
\end{aligned}
$$

where $G_{j}\left(x^{\prime}\right) \equiv g_{j}\left(x^{\prime}\right) g_{j}^{\top}\left(x^{\prime}\right)$,

$$
g_{j}\left(x^{\prime}\right)=-m_{j}\left(x^{\prime}\right) K_{j}^{-1} k_{j}\left(x^{\prime}\right) \quad \text { and } \quad m_{j}^{-1}\left(x^{\prime}\right)=K_{j}\left(x^{\prime}, x^{\prime}\right)-k_{j}^{\top}\left(x^{\prime}\right) K_{j}^{-1} k_{j}\left(x^{\prime}\right) .
$$

Those $O\left(j^{2}\right)$ calculations are a simple consequence of deploying the partitioned inverse equations on the salient elements of Eq. (5), thereby bypassing more expensive $O\left(j^{3}\right)$ ones. Although known for some time in other contexts, Gramacy and Apley chose the acronym ALC to denote the use of that decomposition in local design in order to recognize its first use towards global design of computer experiments by a method called active learning Cohn (1996). That scheme required numerically integrating (7) over the entire design space. Although the localized analog above is simpler because it does not involve an integral, both global and local versions require a computationally intensive search over a large set of candidates $x_{j+1} \in X_{N} \backslash X_{j}(x)$. Speeding up this search is the primary focus of our contribution.

Global emulation, that is predicting over a dense grid of $x$-values, can be done in serial by looping over the $x$ 's, or in parallel since each calculation of local $X_{n}(x)$ 's is independent of the others. This kind of embarrassingly parallel calculation is most easily implemented on symmetric multiprocessor (SMP) machines via OpenMP pragmas. As we demonstrate in Section 5, one can additionally divvy up predictions on multiple nodes of a cluster for very big calculations. Finally, Gramacy and Apley recommend a two-stage scheme wherein local $\hat{\theta}_{n}(x)$ 's are calculated after each local sequential design $X_{n}(x)$ is chosen, so that the second iteration's local designs use locally estimated parameters. This leads to a globally nonstationary model which provides highly accurate predictions under a tight computational budget. The full scheme is outlined algorithmically in Figure 1. It is worth remarking that the scheme is completely deterministic, calculating the same local designs for prediction at $x$, given identical inputs $\left(n\right.$, initial $\theta_{0}$ and data $\left.D_{N}\right)$ in repeated executions. It also provides local uncertainty estimates - a hallmark of any approximation - via Eq. (2) with $D_{n}(x)$, which are organically inflated relative to their full data $\left(D_{N}\right)$ counterparts. Empirically, those uncertainty estimates over cover, as they are perhaps overly conservative. Gramacy and Apley suggest adjustments that can be made to project towards narrower bounds which are closer to their full $N$ counterparts.

\section{GPU computing}

Under NVIDIA's CUDA programming model, work is offloaded to a general purpose GPU device by calling a kernel function - specially written code that targets execution on many hundreds of GPU cores. CUDA has gained wide-spread adoption since its introduction in 2007 and many "drop-in" libraries for GPU-acceleration have been published, e.g., the CUBLAS 
1. Choose a sensible starting global $\theta_{x}=\theta_{0}$ for all $x$.

2. Calculate local designs $X_{n}\left(x, \theta_{x}\right)$ based on ALC, independently for each $x$ :

(a) Choose a NN design $X_{n_{0}}(x)$ of size $n_{0}$.

(b) For $j=n_{0}, \ldots, n-1$, set

$$
x_{j+1}=\arg \max _{x_{j+1} \in X_{N} \backslash X_{j}(x)} v_{j}\left(x ; \theta_{x}\right)-v_{j+1}\left(x ; \theta_{x}\right),
$$

and then update $D_{j+1}\left(x, \theta_{x}\right)=D_{j}\left(x, \theta_{x}\right) \cup\left(x_{j+1}, y\left(x_{j+1}\right)\right)$.

3. Also independently, calculate the MLE $\hat{\theta}_{n}(x) \mid D_{n}\left(x, \theta_{x}\right)$ thereby explicitly obtaining a globally nonstationary predictive surface. Set $\theta_{x}=\hat{\theta}_{n}(x)$.

4. Repeat steps $2-3$ as desired.

5. Output predictions $Y(x) \mid D_{n}\left(x, \theta_{x}\right)$ for each $x$.

Figure 1: Multi-stage approximate local GP modeling algorithm.

library which contains a cublasDgemm function that is the GPU equivalent of the DGEMM matrix-matrix multiplication function from the C BLAS library. Such GPU-aware libraries allow for significant speedups at minimal coding investment, and most use of GPUs for acceleration in statistical applications has been accomplished by replacing calls to CPUbased library functions with the corresponding GPU kernel call from a GPU-aware library (Franey et al., 2012; Eidsvik et al., 2013; Paciorek et al., 2013). This can be an effective approach to GPU-acceleration when the bottleneck in the program lies in manipulating very large matrices, e.g., of dimension $\geq 1000$, as otherwise GPU-aware math libraries can actually be less efficient than CPU ones. In our application, the calculations in Figure 1 involve relatively small matrices by design and therefore do not benefit from this drop-in style approach to GPU-acceleration. Instead, we have developed a custom kernel that is optimized for our relatively small matrices and also carries out many processing steps in a single invocation.

The nuances of our implementation require an understanding of the GPU architecture. In the CUDA computing model, threads are grouped into blocks of up to 1024 threads per block 11 Up to 65535 thread blocks can be instantiated to create the kernel grid, a structure of thread blocks on which a GPU kernel function is invoked ${ }^{2}$ Groups of threads belonging to a given block are simultaneously executed in a warp. All warps derived from a given block are guaranteed to be resident on the same Streaming Multiprocessor (SM) on the GPU device. The number of threads per warp is fixed by the GPU architecture (our cards have 32 threads

\footnotetext{
${ }^{1}$ All values reported here are for CUDA Compute Capability version 2.0 which is the version used in our experiments.

${ }^{2}$ We restrict ourselves to a 1-d grid; more blocks may be instantiated in 2-3d grids.
} 
per warp) and the assignment of threads to warps is controlled by the GPU hardware. The number of blocks that can run simultaneously on a given SM is constrained by the amount of memory and the number of registers required by the threads within each block. The number of SMs and the total number of blocks is fixed by the GPU hardware architecture (our cards have $16 \mathrm{SMs}$, and each can host multiple blocks simultaneously). Assigning multiple blocks to a single SM allows threads from one block to utilize the SM, e.g., perform floating point operations, while threads from another block wait for memory transactions to complete.

Relative to other parallel architectures, GPUs allocate a relatively small amount of memory and registers to each thread. In descending order of access speed, the types of memory utilized for our kernels are registers, shared memory, and local/global memory. Registers and local memory are thread-specific and up to 32768 registers are available to the threads belonging to a given block. Shared-memory (up to 48KB per block) is accessible by all threads belonging to the same block and provides a high-speed location for threads within the same block to communicate with one another and work collectively on data manipulation. Global memory (up to 5GB per GPU device) is accessible by all threads across all blocks, but is an order of magnitude slower than shared-memory and registers. Because all inter-block communication must use global memory, GPU-based applications tend to only achieve high performance on strongly data-parallel algorithms in which work can be cleanly divided across the thread blocks, thereby allowing them to operate independently. For detailed information about parallelism and memory in GPUs, please see Kirk and Wen-mei (2010).

Due to the multiple levels of parallelism, and the different memory types and speeds, constructing kernels can be difficult and, sometimes, counterintuitive. In the remainder of this section, we isolate the calculations from Figure 1 that are best suited to the GPU architecture, describe how those can be implemented on a GPU, and discuss how best to utilize the resulting GPU subroutine in the wider context of global approximate emulation.

\subsection{GPU ALC calculation}

The most computationally intensive subroutine in the local approximate GP algorithm is Step 2(b) in Figure 1; looping over all remaining candidates and evaluating the reduction in variance (6) to find the next candidate to add into the design. Each reduction in variance calculation is $O\left(j^{2}\right)$, and in a design with $N$ points, there are $N^{\prime}=N-j$ candidates. Usually $N \gg j$, so the overall scheme for a single $x$ is $O\left(N n^{3}\right)$, a potentially huge undertaking called for $j=n_{0}, \ldots, n$ for each predictive location $x$. As Gramacy and Apley (2014) point out, it may not be necessary to search over all $N-j$ candidates - searching over a smaller set (say $N^{\prime}=100 n$ ) of NNs can consistently yield the same local design as searching over the full set. However, the resulting $O\left(n^{4}\right)$ search can still represent a considerable computational undertaking, even for modest $n$, when the number of predictive locations is large.

The structure of the evaluations of (6), independent for each of the $N^{\prime}$ candidates, is ideal for GPU computing. Each candidate's calculation can be assigned to a dedicated thread block so long as $N^{\prime}<65535$, i.e. the number of thread blocks. Each of the sequence of $O\left(j^{2}\right)$ operations required for each candidate's calculation (i.e., each block) can be further parallelized across $j$ threads within the designated block so long as $j \leq n<1024$, potentially 
in parallel with many others. Some care is needed to ensure that (a) as many of these independent calculations as possible actually do occur in parallel; (b) threads execute the same instructions on nearby memory locations at the same time for high throughput; (c) there are as few synchronization points as possible; (d) memory transfers to and from the GPU device are minimized; and (e) memory accesses on the GPU are primarily to fast memory locations rather than to high-latency global memory.

Figures 2 and 3 describe our GPU implementation via the data/memory structure and the execution sequence, respectively. In both cases the description is for a particular block and thread within the block, indexed by $b$ and $t$ respectively. The $b$ index selects a candidate $x_{b}$ from a row of the set of remaining candidates $\tilde{X}_{j}(x)=X_{N} \backslash X_{j}(x)$. When the kernel executes, many blocks, i.e., a range of $b$-values, are run in parallel. The number which execute in parallel depends on the size of the problem, $j$ and $N$, and other operating conditions, but we find that it is typically in the hundreds for the problems we've attempted. Within a block, the $t$ index selects a column of a matrix, or an entry of a vector, in order to parallelize the within-block computation. Based on the value of $t$, threads can take different execution paths. However, execution is swiftest when $\approx 32$ threads (the warp size) execute the same sequence of operations on adjacent memory locations. Therefore an effort has been made to avoid divergent execution paths whenever possible.

Figure 2 describes the inputs/outputs (first two tables) and the working memory (last two) of the GPU kernel. The right-hand columns of the tables describe variables in terms of quantities in Eq. (6). Note that some are reused. It also indicates what type of memory the variable is stored in. Initially, all non-scalar inputs reside in slow global memory. Parts of global memory that are frequently accessed by the block, $b$, are copied into that block's shared memory. Shared memory locations which are repeatedly accessed by particular threads, $t$, within a block use temporary register storage. No local memory is required for our kernels. As a visual queue we use a $t$ subscript to distinguish between a register quantity indexing a particular value of a shared memory array. For example, $k_{t}$ is used to calculate what will eventually reside in $k[t]$, the $t^{\text {th }}$ indexed shared memory mapping pointed to by $k$. Eventually, $k$ will store $k_{j}\left(x_{b}\right)$, a $j$-vector, and will later be reclaimed to store $K_{n}^{-1} k_{j}\left(x_{b}\right)$.

Several of the steps outlined in Figure 3 require more detailed explanation. Notice that Steps $2 \& 6$ assume an isotropic Gaussian correlation function. Simple modification would accommodate another family and/or a separable version via a vectorized $\theta$ parameter. In two places, a sequence of two synchronized steps $(3 \rightarrow 4$ and $6 \rightarrow 7)$ calculate the scalar value(s) of a quadratic form by first having each thread, asynchronously, fill a particular entry in a $j$-vector, and then sum its elements via a reduction. Reductions are a way to get multiple threads to work simultaneously towards calculating something that is more natural serially, like a sum. Our implementation, abstracted as "sum.reduce $(t, v)$ " for thread $t$ 's contribution to calculating the sum of the vector $v$, uses the logarithmic version described on the SHARCNET (2012) pages, which makes use of $\lfloor j / 2\rfloor$ threads. Since more than half of the threads are idle in this reduction, we implemented our own bespoke version (employing the idle threads) for the two simultaneous reductions required by Step 7, which led to a $10 \%$ speedup compared to two separate reductions. [See Appendix A.] 
The thread is indexed by $t$, and the block by $b$.

Scalar inputs stored in registers:

variable description

$j$ integer number of rows in the current local design $X_{j}(x)$

$\theta$ double precision lengthscale parameter $\theta$

$\eta$ double precision nugget parameter $\eta$

$N$ the number of rows $(N-j)$ in the candidate matrix $\tilde{X}=X_{N} \backslash X_{j}(x)$

$p$ integer number of columns in $X_{j}(x)$ and $\tilde{X}$

Double-precision input (and output) arrays stored in global memory:

\begin{tabular}{r|l} 
variable & description \\
\hline$X$ & row-wise flattened $X_{j}(x)$, a $j \times p$ matrix \\
$K^{-1}$ & row-wise flattened $K^{-1}$, a $j \times j$ matrix \\
$\tilde{X}$ & row-wise flattened $X_{N} \backslash X_{j}(x)$, a $(N-j) \times p$ matrix \\
$h$ & covariances $K(x, X)$ between $x$ and rows of $X$, an $n$-vector \\
$\Delta$ & an $N-j$ vector containing the output of Eq. 6
\end{tabular}

Double-precision working memory scalars stored in registers:

\begin{tabular}{r|l} 
variable & eventual contents via analog in Eq. \\
\hline$m^{-1}$ & $m_{j}^{-1}$, identical for all threads in block $b$ \\
$k_{t}$ & $\left(k_{j}(x)\right)_{t}$, the $t^{\text {th }}$ element of $k_{j}(x)$ \\
& later re-used for the $t^{\text {th }}$ entry of $K_{j}^{-1} k_{j}\left(x_{b}\right)$ \\
$g_{t}$ & $\left(g_{j}\left(x_{b}\right)\right)_{t}$, the $t^{\text {th }}$ element of $g_{j}\left(x_{b}\right)$ \\
$\ell_{t}$ & $K\left(x_{b}, x\right)$, identical for all threads $t$ in block $k$ \\
& later re-used for the $t^{\text {th }}$ entry of $G_{j}^{-1}\left(x^{\prime}\right) m_{j}^{-1}\left(x_{b}\right) k_{j}(x)$
\end{tabular}

Double-precision working memory arrays stored in shared memory:

\begin{tabular}{r|l} 
variable & eventual contents via analog in Eq. $(6)$ \\
\hline$x_{b}$ & a $p$-vector: the $b^{\text {th }}$ candidate/row of $X$ \\
$k$ & $\begin{array}{l}k_{j}(x), \text { a } j \text {-vector; } \\
\text { later re-used for element-wise product of } k_{j}\left(x_{b}\right) \text { and } K_{j}^{-1} k_{j}\left(x_{b}\right) \\
g \\
\ell\end{array}$ \\
$\begin{array}{l}g_{j}\left(x_{b}\right), \text { a } j \text {-vector } \\
\text { a } j \text {-vector with element-wise product of } k_{j}(x)^{\top} \text { and } K_{j}^{-1} k_{j}\left(x_{b}\right) ; \\
\text { later re-used for product of } k_{j}(x)^{\top} \text { and } G_{j}^{-1}\left(x^{\prime}\right) m_{j}^{-1}\left(x_{b}\right) k_{j}(x)\end{array}$
\end{tabular}

Figure 2: Inputs, outputs and working memory used by the GPU kernel computing Eq. (6). 
Recall that $t$ indexes the thread and $b$ indexes the block.

Each enumerated set of instructions is implicitly followed by a thread synchronization.

1. \% Copy the $b^{\text {th }}$ candidate (row of $\tilde{X}$ ) into faster shared memory.

if $(t<p)$ then $x_{b}[t] \leftarrow \tilde{X}_{b}[b \times p+t]$

2. $\%$ Calculate $K_{j}\left(x_{b}, x\right)$.

$k_{t} \leftarrow 0$

for $(i \in\{1: p\})$ do $k_{t} \leftarrow k_{t}+\left(x_{b}[i]-X[t \times p+i]\right)^{2}$

$k[t] \leftarrow \exp \left\{-k_{t} / \theta\right\}$

3. $\%$ Initialize $g_{j}\left(x_{b}\right)$ with $K_{j}^{-1} k_{j}\left(x_{b}\right)$, and prepare $k_{j}\left(x_{b}\right)^{\top} K_{j}^{-1} k_{j}\left(x_{b}\right)$.

$g_{t} \leftarrow 0$

for $(i \in\{1: j\})$ do $g_{t} \leftarrow g_{t}+k[i] \times K^{-1}[i \times j+t]$.

$\ell[t] \leftarrow g_{t} \times k[t]$

4. $\%$ Complete the dot product $k_{j}\left(x_{b}\right) \cdot K_{j}^{-1} k_{j}\left(x_{b}\right)$.

$\ell[0] \leftarrow \operatorname{sum} . r e d u c e(t, \ell)$

5. $\%$ Calculate $\mu_{j}^{-1}\left(x_{b}\right)$, and finish $g_{j}\left(x_{b}\right)$.

$m^{-1} \leftarrow 1.0+\eta-\ell[0]$

$g[t] \leftarrow g_{t} / m^{-1}$

$\%$ Without syncing threads, calculate $K_{j}\left(x, x_{b}\right)$ and initialize the output array. ${ }^{*}$

$k_{t} \leftarrow 0$

for $(i \in\{1: p\})$ do $k_{t} \leftarrow k_{t}+\left(x_{b}[i]-x[i]\right)^{2}$

$\Delta[b] \leftarrow \exp \left\{-k_{t} / \theta\right\}$

6. \% Prepare $k_{j}(x)^{\top} G_{j}^{-1}\left(x_{b}\right) k_{j}(x)$ and $k_{j}^{\top}(x) g_{j}\left(x_{b}\right)$.

$\ell_{t} \leftarrow 0$ for $(i \in\{1: j\})$ do $\ell_{t} \leftarrow \ell_{t}+g[t] \times g[i] \times \mu_{t}^{-1}$

$\ell[t] \leftarrow \ell_{t} \times h[t]$

$k[t] \leftarrow h[t] \times g[t]$

7. $\%$ Complete the dot products $k_{j}(x) \cdot G_{j}^{-1}\left(x^{\prime}\right) m_{j}^{-1}\left(x_{b}\right) k_{j}(x)$ and $k_{j}(x) \cdot K_{j}^{-1} k_{j}\left(x_{b}\right)$.

$\ell[0] \leftarrow$ sum.reduce $(t, \ell)$

$k[0] \leftarrow \operatorname{sum} . \operatorname{reduce}(t, k)$

8. \% Wrapping up Eq. (6).*

$\Delta[b] \leftarrow \ell[0]+2.0 \times k[0] \times \Delta[b]+\Delta[b]^{2} / \mu^{-1}$

Figure 3: Psuedocode for the GPU kernel computing Eq. (6). Comments are indicated by lines beginning with "\%"; those followed by "** superscripts indicate that the following commands need only be executed on one thread, e.g., thread $t=0$. 
Step 3 is the most computationally intensive, since it involves accessing $j$ items stored in global memory, the $t^{\text {th }}$ column of $K^{-1}$. There is one other $j$-loop (Step 6), but it accesses faster shared memory. Staging the $t^{\text {th }}$ column of $K_{j}^{-1}$ in shared memory does not lead to a faster implementation since multiple accesses of this data are not required within the block. By contrast, we copy a row of $\tilde{X}$ in to shared memory (Step 1) since it is reused (Step 5), thereby avoiding multiple transactions on global memory in this phase of the algorithm. We remark that it is very important to work column-wise with $K^{-1}$ as opposed to row-wise to ensure coalescence in memory transactions. Working column-wise allows warps of threads to access adjacent memory locations storing $K^{-1}$. Working row-wise, i.e., accessing $K[t \times j+i]$, gives the same answer (because the matrix is symmetric) but is about $j$-times slower.

Finally, we remark that the output, $\Delta$, is not normalized. A final step, multiplying by $\psi /(j-2)$, is required to match the expression in Eq. (6). This can be done as a CPU post-processing step, although it is slightly faster on the GPU. In Figure 3 .

9. \% Normalize by the global variance estimate. $\Delta[b] \leftarrow \psi \Delta /(j-2)$.

Observe that this is not actually required to find the argmax in Step 2b of Figure 1.

\subsection{GPU-CPU full GP approximation}

The GPU kernel described above implements Steps 2a and 2b in Figure 1. Here we discuss how it can be best situated within the outer loop(s), ultimately being applied over all predictive locations $x \in \mathcal{X}$. The simplest option is to serialize: simply calculate for each $x$ in sequence, one after another. Within that loop, iterate over $j=n_{0}, \ldots, n$, performing the required CPU calculations amidst GPU kernel calls to calculate Eq. (6). We show in Section 4 that this leads to significant speedups compared to a serial CPU-only implementation. But it makes for an inefficient use of a multiplicity (i.e., 1-CPU and 1-GPU) of computing resources. The CPU is idle while the GPU is working, and vice versa.

Both inefficiencies are addressed by deploying a threaded CPU version identical to the original one advocated by Gramacy and Apley (2014), i.e., using OpenMP. The difference here is that speedups are attained even in the case of a single CPU core because while one CPU thread is waiting for a GPU kernel to finish other CPU threads can be performing CPU operations and/or queuing up the next GPU calculations. Having a small backlog of GPU kernels waiting for execution is advantageous because it means the next kernel will start immediately after the current one finishes.

There are diminishing returns for increasing numbers of CPU threads as they compete for resources on both the CPU and GPU. There would eventually be negative returns due to inefficiencies on the CPU (too many context switches) or GPU (not enough memory to queue executions). This latter concern is very unlikely though since, e.g., our device has more than 5GB of global memory. When $N^{\prime}<65535$ and $j \leq n<1024$, i.e., the block and thread constraints, we can still queue quite a few kernels. In typical approximations $N^{\prime}$ and $n$ are an order of magnitude smaller and we find that (marginal) speedups are still observed 
when there are more than 4 CPU threads per CPU core (in the 1-GPU case).

Obviously, when there are multiple CPUs and/or multiple GPUs, CPU threading is essential lest the duplicated resources remain untapped. We assume here that all GPUs are identica $\mathrm{I}^{3}$ and, so long as the CPU threads spread the kernels roughly equally amongst GPUs, no further load balancing considerations are required (Hagan, 2011). Given a fixed number of GPUs (including zero for a CPU-only version) we find a nearly linear speedup as CPU cores (with one thread each) are added. Multiple threads-per-CPU core can help, although only marginally as the number of cores increases, and only if there is at least one GPU. For example, we will show that 32 threads with 16 cores and one or two GPUs is marginally faster than using 16 threads - one per core. As GPUs are added the initial benefits are substantial, especially when there are few CPUs. In that case it again makes sense to have more threads than CPU cores.

\section{Empirical results for the new GPU version}

In this section we borrow the 2-d data and experimental apparatus of Gramacy and Apley (2014). This allows us to concentrate on timing results only - the accuracy, etc., of the resulting predictions are identical to those reported in that paper. Our discussion is broken into two parts: first focusing on the ALC calculations in isolation; then as applied in sequence to build up local designs for many input locations, independently (and in parallel). The node we used contains two NVIDIA Tesla M2090 GPU devices with 5GB of global memory and the L1 cache option is set to prefer shared-memory (giving 48KB per block). It has dual-socket 8-core 2.6 GHz Intel Sandy Bridge Xeons with 32GB of main memory.

\subsection{GPU calculations}

Here we study the performance of GPU ALC calculations [Section 3.1] relative to the oneCPU-only alternative, beginning with Figure 4 which summarizes the result of an experiment set up in the following way. We focus on a single reference location, $x$, the value of which is not important as the timing results are the same for any $x$. We consider $N^{\prime}=60 \mathrm{~K}$, which is close to the maximum number of blocks, with one block per candidate. The only thing that varies is the local design size $n$, from $n=16$ to $n=512$ (in steps of size 4 ). All of the required correlation matrices, etc., are presumed to be calculated in advance (conditional on sub-designs $D_{n}(x)$, and candidates $\left.\tilde{X}\right)$. The time needed to build these is not included in the comparison, as they are required as inputs by both CPU and GPU methods. The extra time needed to copy data from CPU to GPU is, however, included in the GPU timings.

The left panel of the figure shows log timings separately for the CPU and GPU; whereas the right panel shows the relative speedup offered by the GPU obtained by dividing the CPU time by the corresponding GPU one. We observe the following. The speedups range between 20x and 75x, with more modest speedups for small $n$ owing to fewer economies of scale. Two explanations are: (a) initiating data transfers to the GPU are relatively expensive

\footnotetext{
${ }^{3}$ Modern multi-core CPUs are always identical when in the SMP configuration.
} 

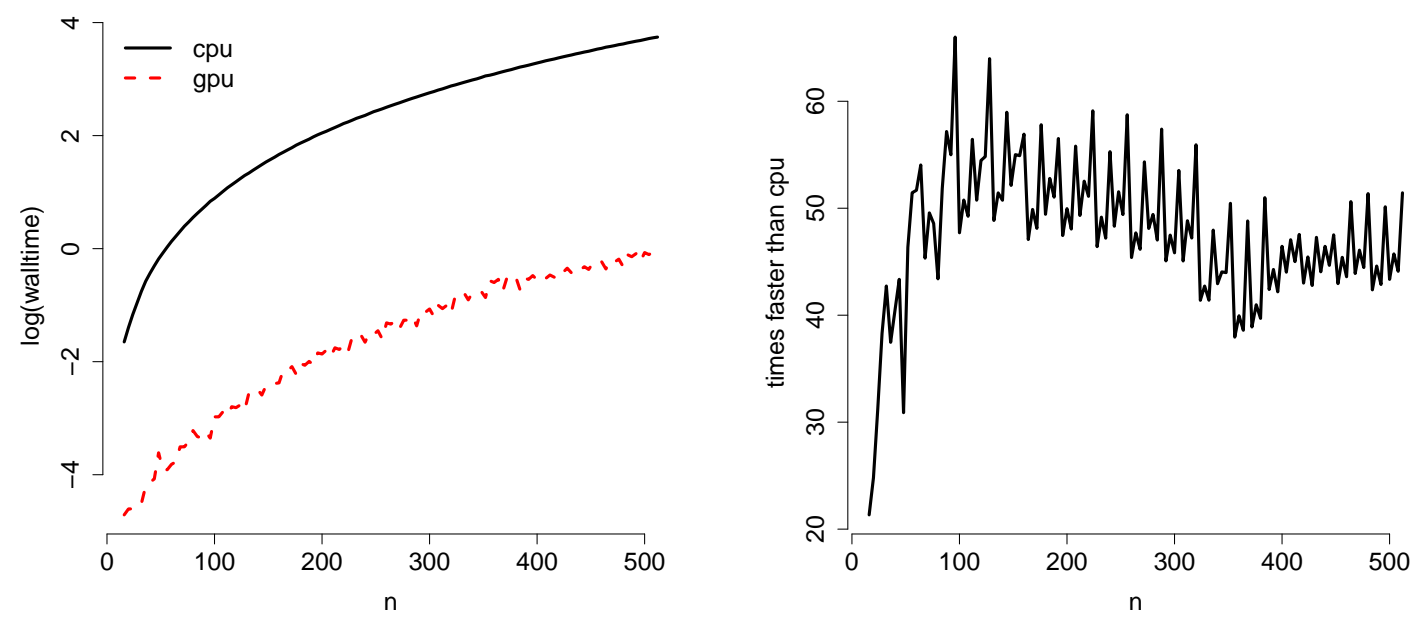

Figure 4: Comparing CPU-only and GPU-only wall-clock timings for the ALC calculation at each of $n_{\text {cand }}=60000$ candidate locations for varying $n$, the size of the local design. It is assumed that the relevant covariance structure(s) are precomputed, so these are not incorporated into the timing results. The left plot shows absolute performance on a log scale; the right plot makes a relative comparison via ratios of times.

when only a small amount of data is sent/received; and (b) when $n$ is small the number of GPU threads-per-block (also $n$ ) is low, which results in low GPU throughput. We note that it may be possible to increase performance in the second case, for low block index $b$, by implementing more complex thread allocation logic to increase the number of threads-perblock. However, we favor a direct mapping that leads to a clear implementation over a more complex but potentially more performant solution.

Observe [Figure 4, right] that the time series of GPU compute times is periodic, which is a consequence of the GPU architecture. There are several factors contributing to this phenomena. Executions where $n$ is a multiple of the warp size, i.e., $n=32 k$, tend to be faster, on average. Our reduction scheme (for dot products, etc.) is fastest when $n$ is a power of 2 . There is a sweet spot near $n=128$, a power of 2 and multiple of 32 , with diminishing returns thereafter due to the quadratically growing $K_{n}^{-1}$ that must be transferred to the GPU over the relatively slow PCIe bus.

\subsection{Full GP approximations via CPU and GPU}

Here we study how the entire local design scheme, deploying extensive ALC search as a subroutine, compares under GPU versus CPU when calculated for a dense set of predictive localtions $x$ in a global prediction exercise. As in Section 4.1, we primarly vary the approximation fidelity via the local design size, $n$.

A quick profiling of a CPU-only version, varying $n$ and $x$, reveals that ALC computations 
represent the majority of compute cycles, at 50-98\%. That wide range arises due the relative amount of ALC work required compared to other calculations. For example, each iteration $j$ requires CPU routines to update the GP correlation structure. At the end, when $j=n$, MLE calculations may also be invoked. Both can require a relatively substantial number of cycles depending on the value of $j, n$, and the quality of initial $\theta_{x}$ values, with bad ones leading to more likelihood evaluations to find the MLE. As $j, n$, and $N^{\prime}$ are increased, leading to a higher fidelity approximation, the computational demands increase for ALC relative to the other CPU routines, leading to more impressive speedups with a GPU implementation.

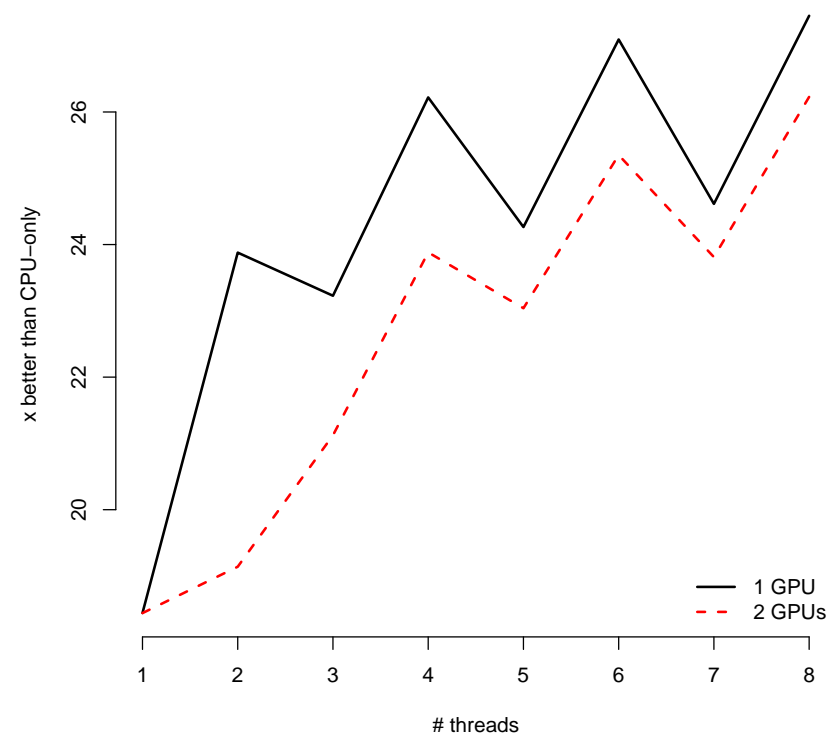

Figure 5: Speedups from GPU version(s) for varying CPU threads on a 1-core CPU.

To start things off, Figure 5 compares a CPU-only version to one leveraging one- and two-GPU ALC calculations. The metric shown on the $y$-axis is a ratio of the wall-clock execution times obtained while predicting at $\sim 10 \mathrm{~K}$ locations, with $n=50$ and using $N^{\prime}=$ 1000 NN candidates. The numerators in that ratio are times calculated from the reference implementation, a single CPU core without GPU(s). The denominators come from each CPU-GPU competitor, with one or two GPUs respectively, so that we may interpret the ratio as a factor of improvement over a single-CPU-core-only implementation. (Otherwise the setup is identical to the previous subsection.) The $x$-axis varies the number of OpenMP CPU threads, where each thread works on a different local predictive location $x$, as described briefly in Section 2.2. Threaded computing does not benefit a single-CPU-core-only version. However, since GPUs can execute in parallel with other CPU calculations, the entire scheme benefits from having multiple CPU threads because they can asynchronously queue jobs on the GPU devices. The trend is that speeds increase, with diminishing returns, when the number of threads is increased. Notice how odd numbers of threads are sub-optimal, which 
is a peculiarity of the particular GPU-CPU architecture on our machine. Also, notice how the 2-GPU setup is marginally slower than the 1-GPU one, suggesting that the single CPU core is not able to make efficient use of the second GPU device.

The previous example illustrates how CPU-only and CPU/GPU schemes might compare on an older-model laptop or desktop connected to a modern GPU. These days even laptops are multi-cored (usually two cores), and most modern research workstations have at least four cores. It is not uncommon for them to have up to sixteen. Therefore we next factor SMP-style parallelization into the study. In some ways this leads to a fairer CPU vs. GPU comparison, since GPUs are technically multi-core devices (albeit with very simple cores). Figure 6
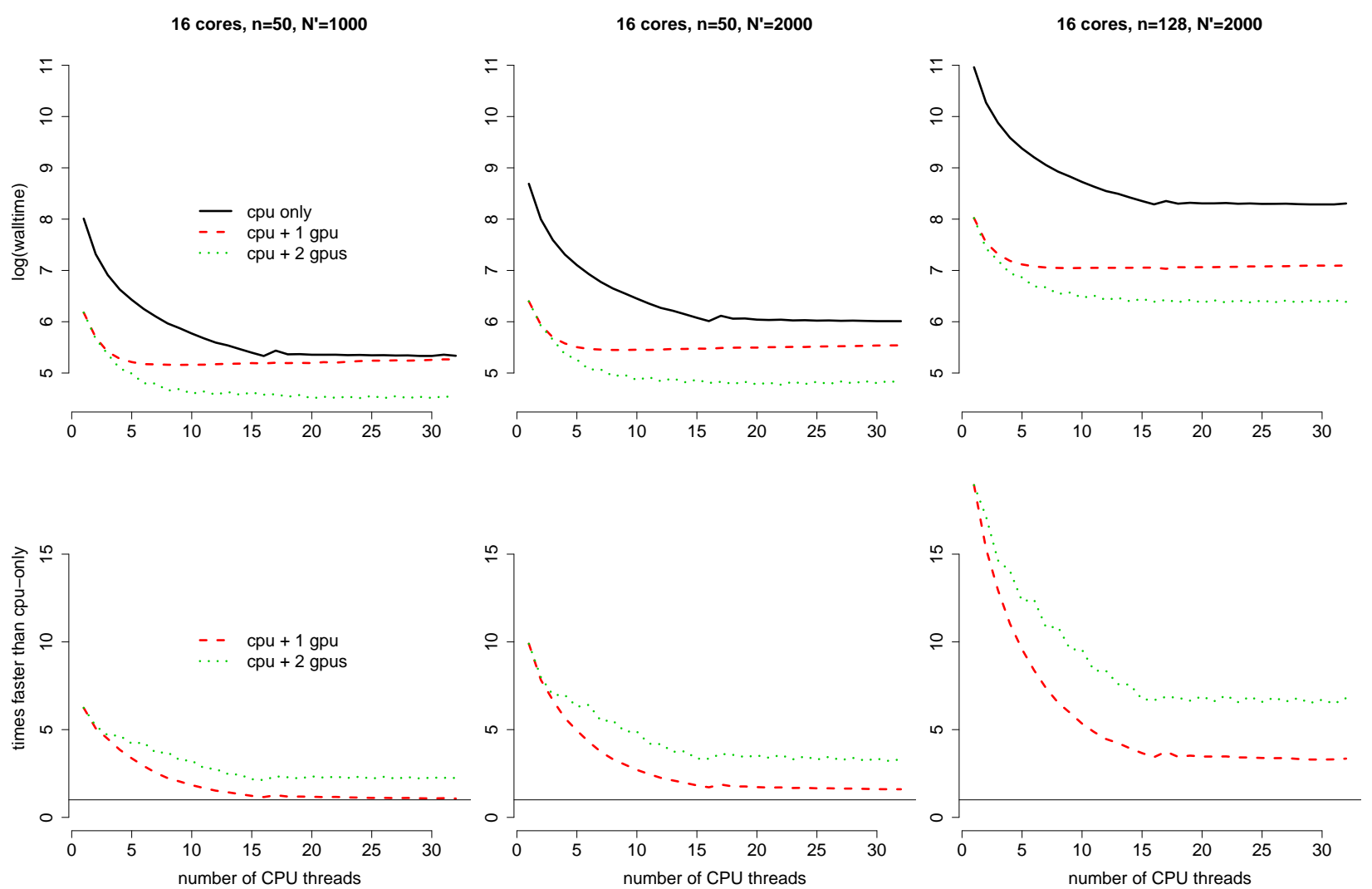

Figure 6: Comparing full global approximation times for a $\sim 40 \mathrm{~K}$-sized design, and a $\sim 10 \mathrm{~K}$ predictive locations, under various CPU/GPU configurations and approximation fidelities (larger $n$ and/or $N^{\prime}$ ). The top row makes absolute comparisons via log compute times; the bottom row makes a relative comparison to CPU-only compute times via a ratio. The solid horizontal line is at one on the $y$-axis.

summarizes the result of an experiment utilizing up to 16 cores and 2 GPUs. Problem sizes are varied along the columns of the figure $\left(\left(n=50, N^{\prime}=1000\right),\left(n=50, N^{\prime}=2000\right)\right.$, $\left(n=128, N^{\prime}=2000\right)$ ) and a number of OpenMP CPU threads and GPUs is varied in each plot. The top row in the figure shows log timings, whereas the bottom row shows times 
relative to the CPU-only calculation, via the same ratio used in Figure 5.

First consider the results obtained for a single CPU thread. The figure shows that the ALC GPU computation yields between a 6x and 20x speedup, with the better ratios obtained as the fidelity of the approximation increases. Note that the 1-GPU and 2-GPU results are the same in this case - a single CPU thread cannot make effective use of multiple GPUs.

When multiple OpenMP CPU threads are in use the compute times steadily decrease as more processors are added. For example, considering the CPU-only results, the ratio of the 1-CPU time to the 16-core CPU time is 14.5 for all three problem sizes, which suggests very good efficiency (the best we could hope for is $16 \mathrm{x}$ ). In the case of the first column, the 16 CPU thread solution is faster than the 1-CPU-thread version interfacing with a GPU. (In fact it is better up to about $5 \mathrm{CPU}$ threads for the GPU). Using $16 \mathrm{CPU}$ threads performing CPU-only calculations is almost as good as allowing those same 16 threads to queue jobs on a single GPU, which is creating a bottleneck. The results for two GPUs are much better, and we would expect the relative timings to be even better with more GPUs.

We conclude that both SMP and GPU paradigms are helpful for calculating the local GP approximation. Their combined efforts, compared to using a single CPU alone, represent a $33 \mathrm{x}, 50 \mathrm{x}$, and 100x, improvement in wall-clock time on the three problem sizes respectively. If only one option were made available, single CPU-only or single GPU+CPU, the latter is clearly preferable (giving 6-20x speedups). However, adding on multiple CPU threads can lead to a 2.6x speedup in the 1-GPU setting, and about 5x in the 2-GPU case, for all three problems. Finally, augmenting a 16 CPU-only setup with 2 GPUs gives a $2.2 x, 3.3 x, 6.7 x$ speedups respectively.

We noticed in this latter case, with 16 CPUs and 2 GPUs, that only 5/16 of CPU capability was being utilized with so much of the computation being off-loaded to the GPUs. We found that when this happens it is possible to get a further $1 / 4$ reduction in wall-clock times by creating new CPU threads (10 or so) to do CPU-only ALC calculations alongside the GPU ones. Some pilot tuning is needed to get the load balancing of CPU v. GPU calculations right. Figure 6 can serve as a guide, starting with an 80/20 GPU/CPU split for the lowest fidelity case, increasing 85/15 and 90/10 as the fidelity, and thus relative speedup obtained from the GPU, is increased.

\section{$5 \quad$ Big computer emulation}

Here we demonstrate a three-level cascade of parallelism towards approximate GP emulation on very big computer experiment data. The first two levels are OpenMP-based and CUDAbased, using CPUs and GPUs respectively, on a single compute node. The third level is a cluster, allowing simultaneous use of multiple nodes. For this we use the simple network of workstations model implemented in the snow package (Tierney et al., 2013) for R, which only requires a simple wrapper function to break up the predictive locations $\mathcal{X}$ into chunksallowing each to be processed on a separate node via clusterApply - and then to combine 
the outputs into a single object 4

We remark that in the case of a single node with multiple cores, our use of snow accomplishes something very similar to an OpenMP SMP parallelization. However, given a choice the latter is faster than the former since establishing a cluster requires starting multiple copies of $\mathrm{R}$, sending copies of the data to each, and then combining the results. Also, our focus here is primarily on timing results, reminding readers that fidelity/computational demands are tightly linked with emulation accuracy. In the case of our second example, Gramacy and Apley (2014) already illustrated how a relatively thrifty approximation can provide more accurate predictions compared to modern alternatives in a fraction of the time.

\subsection{Langley Glide-Back Booster}

Our first example is a real computer experiment for a re-usable rocket booster called the Langley Glide-Back Booster. The computer model, developed at NASA, involves computational fluid dynamics (CFD) codes that simulate the characteristics of the booster as it re-enters the atmosphere - modeling outputs such as lift as a function of inputs such as speed, angle of attack, and side-slip angle. For more details of the experiment, including how the emulator can benefit from a nonstationary/localized modeling capability due to abrupt dynamical transitions at speeds near the sound barrier, see Gramacy and Lee (2009). The design ${ }^{5}$ has $N=37908$ 3-dimensional input configurations, and six outputs but we only consider the first one, lift, here. The design is gridded to be dense in the first input, speed, and coarse in the last, side slip angle. We consider using the local GP approximation method to interpolate the lift response onto a regular grid that is two-times more dense in the first input, and three times more dense in the second two. That gives a predictive grid of size $|\mathcal{X}|=644436$.

Our setup here mimics the apparatus described in Section 4.2 , using 4 identical compute nodes, each having 16 cores and 2 GPUs. We establish 16 OpenMP CPU threads queuing GPU ALC calculations on both GPUs, and 12 further OpenMP CPU threads performing CPU-only ALC calculations, initially allowing the GPUs to take $80 \%$ of the ALC work. The snow package distributes an equal workload to each of the four nodes. The ALC searches are over $N^{\prime}=1000 \mathrm{NN}$ candidate locations, starting at $n_{0}=6$ and ending at $n=50$, i.e., following the left-most panel in Figure 6. The wall-clock time for the full emulation was 21 minutes. By way of comparison, a single CPU-only version (but fully utilizing its 16 cores) takes 235 minutes (4 hours) and fully using all CPU cores on all four nodes takes about 58 minutes ( 1 hour). Therefore the GPUs yield about a $4 \mathrm{x}$ speedup, which is a little better than the $2 \mathrm{x}$ speedup indicated in the bottom-left panel Figure 6 .

A higher fidelity search with $N^{\prime}=2000$, mimicking the middle panel of Figure 6 except the CPU/GPU load was beneficially re-balanced so that GPUs do $90 \%$ of the work, took 33 minutes in the full (4x 2-GPU/16-CPU) setting. A single CPU-only version (16 cores)

\footnotetext{
${ }^{4}$ The built-in parallel package can also be used instead of snow. In our setup, cluster nodes are allocated via SLURM, an open-source Linux scheduler, and are connected by an Infiniband FRD10 fabric.

${ }^{5}$ The version of the data we consider here is actually the output of Gramacy \& Lee's emulation of computer simulations adaptively designed to concentrate more runs near speeds of Mach one, i.e., at the sound barrier, using a partition-based non-stationary model.
} 
takes 458 minutes ( $\sim 8$ hours) and using all four nodes takes 115 minutes ( $\sim 2$ hours). So the GPUs yield a 4x speedup, which is in line with the bottom-middle panel of Figure 6 . Increasing the fidelity again to $N^{\prime}=10 \mathrm{~K}$, and re-balancing the load so that GPUs do $95 \%$ of the ALC work by allocating 12 extra CPU threads to do the rest, takes 112 minutes on all 4x 2-GPU/16-CPU nodes, representing a more than 5x speed-up compared to the $4 \mathrm{x} 16$-CPU (i.e., no GPU) version. Alternatively, keeping $N^{\prime}=2000$ but increasing the local design size to $n=128$, mimicking the right column of Figure 6 except with GPUs again doing $95 \%$ of the ALC work, takes 190 minutes, representing an almost 6x speedup.

\subsection{A one-hour supercomputing budget}

We wrap up with a search for the largest emulation possible on the resources available to us. For data generation we chose the borehole function (Worley, 1987; Morris et al., 1993) which provides a familiar benchmark. It has an 8-dimensional input space, and our use of it here follows directly from Gramacy and Apley (2014) who copied the setup of Kaufman et al. (2012); more details can be found therein.

Table 1 summarizes the timings and accuracies of designs from size $N=1000$ to just over $N=1 \mathrm{M}$, stepping by factors of two. To keep things simple, the predictive set size is taken to match the design size $(|\mathcal{X}|=N)$, but note that they are different random (Latin hypercube) samples. We allowed the fidelity of the approximation to increase with $N$ along a schedule that closely matches settings that have worked on similarly sized problems. Specifically, we started with $\left(n=40, N^{\prime}=1000\right)$ for the smallest problem $(N=1000)$, and each time $N$ doubled we increased $n$ additively by two and $N^{\prime}$ multiplicatively by 1.5 . The left panel of the table shows results from a 96-node CPU cluster, where each node has 16 cores. The middle panel shows results from a 5 -node GPU/CPU cluster, where each node has 2 GPUS and 16 cores. Unfortunately, the infrastructure we had access to did not allow CPU and GPU/CPU nodes to be mixed. The final panel shows the speedup-factor from the GPU nodes assuming we had 96 instead of five.

On the CPU nodes, over a million inputs and outputs can be processed in under an hour, $\sim 100 \mathrm{~K}$ in about a minute, and $\sim 10 \mathrm{~K}$ in about two seconds. The GPU/CPU cluster has a little less than half of the capacity, processing half-a-million points in just over an hour. Assuming we had more GPU/CPU nodes, the final column suggests that the GPUs make the whole execution 2.5-4.5x faster on these problems. Notice that these efficiencies decrease and then increase again as fidelity is increased. The initial high efficiencies are actually due to inefficiencies in the snow execution: 96 cores is overkill for problems sized in the few thousands, requiring too much of a communication overhead between master and slave nodes. Whereas the latter high efficiencies are due to improvements in GPU throughput for larger problems. Finally, notice that accuracy (out-of-sample MSE) is steadily improving as fidelity increases. By way of comparison, Gramacy and Apley (2014) showed that with $N \in\{4000,8000\}$ the approximations were at least as accurate as those in Kaufman et al. (2012) with less than $1 \%$ of the computing effort.

However, comparisons based on accuracy in this context are at best strained. In cases when each method can execute fast enough to perform a full analysis (e.g., limiting to 


\begin{tabular}{rrr|rr}
\multicolumn{1}{|c|}{$96 \mathrm{x}$ CPU } \\
\hline$N$ & $n$ & $N^{\prime}$ & seconds & $\mathrm{mse}$ \\
\hline 1000 & 40 & 100 & 0.48 & 4.88 \\
2000 & 42 & 150 & 0.66 & 3.67 \\
4000 & 44 & 225 & 0.87 & 2.35 \\
8000 & 46 & 338 & 1.82 & 1.73 \\
16000 & 48 & 507 & 4.01 & 1.25 \\
32000 & 50 & 760 & 10.02 & 1.01 \\
64000 & 52 & 1140 & 28.17 & 0.78 \\
128000 & 54 & 1710 & 84.00 & 0.60 \\
256000 & 56 & 2565 & 261.90 & 0.46 \\
512000 & 58 & 3848 & 836.00 & 0.35 \\
1024000 & 60 & 5772 & 2789.81 & 0.26 \\
\hline
\end{tabular}

\begin{tabular}{rr}
\multicolumn{2}{c}{$5 \times 2$ GPUs } \\
\hline seconds & mse \\
\hline 1.95 & 4.63 \\
2.96 & 3.93 \\
5.99 & 2.31 \\
13.09 & 1.74 \\
29.48 & 1.28 \\
67.08 & 1.00 \\
164.27 & 0.76 \\
443.70 & 0.60 \\
1254.63 & 0.46 \\
4015.12 & 0.36 \\
13694.48 & 0.27 \\
\hline
\end{tabular}

$\begin{array}{r}\frac{\mathrm{CPU}}{5 \cdot \mathrm{GPU} / 96} \\ \hline \text { efficiency } \\ \hline 4.73 \\ 4.26 \\ 2.79 \\ 2.66 \\ 2.61 \\ 2.87 \\ 3.29 \\ 3.63 \\ 4.01 \\ 4.00 \\ 3.91 \\ \hline\end{array}$

Table 1: Timings and out-of-sample accuracy measures for increasing problem sizes on the borehole data. The "mse" columns are mean-squared predictive error to the true outputs on the $|\mathcal{X}|=N$ locations from separate runs (hence the small discrepancies between the two columns). Both CPU and GPU nodes have 16 CPU cores. So the "96x CPU" shorthand in the table indicates $1536 \mathrm{CPU}$ cores.

$n<10000$ ), we've observed (based on comparisons like the ones above) accuracies that are strikingly similar across a wide swath of comparators. We think it is reasonable to suggest that would remain true for larger problem sizes, although this is nearly impossible to verify. Often the largest runs reported by authors are on proprietary data, and some involve proprietary library routines, which makes reproducibility difficult. For example, the largest problem entertained by Kaufman et al. (2012) was a cosmology example with $(N=$ $20000,|\mathcal{X}|=80000)$, but timing information was not provided and the data is not publicly available to our knowledge. Paciorek et al. (2013) entertained $(N=67275,|\mathcal{X}|=55379)$, but again without timing information or public data. Therefore, we conclude that our method is at worst a worthy competitor relative to these alternatives, but offering the potential for similar emulation quality on problems that are several orders of magnitude larger.

\section{Discussion}

The local GP approximation of Gramacy and Apley (2014) swaps a large problem for many small independent ones. We show in this paper how those many small problems can be solved on a cascade of modern processing units. We think this is particularly timely research. Many modern desktops have multiple cores and (sometimes) multiple GPUs, and many modern "supercomputers" are not much more than enormous clusters of high-end multi-core desktops and GPUs.6 Our primary focus was on a GPU accelerated version of a key subroutine in

\footnotetext{
${ }^{6}$ Some really modern supercomuters are essentially clusters of GPUs, with very little CPU computing capability, although we did not have access to such a setup for the empirical work in this paper.
} 
the approximation, allowing a faster execution at lower cost. Although results have emerged casting doubt on some of the speed claims made in scientific computing contexts for GPUs (e.g., Lee et al., 2010), it is still the case that, penny-for-flop, GPUs are cheap. Therefore, its proportion of available flops will continue to grow relative to CPUs for some time to come.

We take a different tack to the use of GPUs for GP computer emulation compared to other recent works, which primary offload large matrix calculation to GPUs. As we show, the combined effects of approximation and massive parallelization can extend GP emulation to problems at least an order of magnitude larger than what is currently possible. We note that others have had similar success parallelizing non-GP models for computer emulation. For example, Pratola et al. (2013) parallelized the Bayesian additive regression trees (BART) method using the message passing interface (MPI) and report handling designs as large as $N=7 \mathrm{M}$ using hundreds of computing cores. Such efforts will likely remain in vogue so long as computing resources continue to grow "out" (with more nodes/cores, etc.) faster than they grow "up", which will be for quite some time to come.

\section{Acknowledgments}

This work was completed in part with resources provided by the University of Chicago Research Computing Center. Many thanks to Matt Pratola for comments on an early version.

We are grateful for valuable comments from two referees and an associate editor during the formal review process.

\section{A Double reduction CUDA code}

The CUDA GPU kernel sumBoth assumes that both inputs, d_data1 and d_data2 have length $\mathrm{n}$, and the call is sumBoth $\langle<1, \mathrm{n}\rangle\rangle$ (d_data1, d_data2) so that blockDim. $\mathrm{x}=\mathrm{n}$.

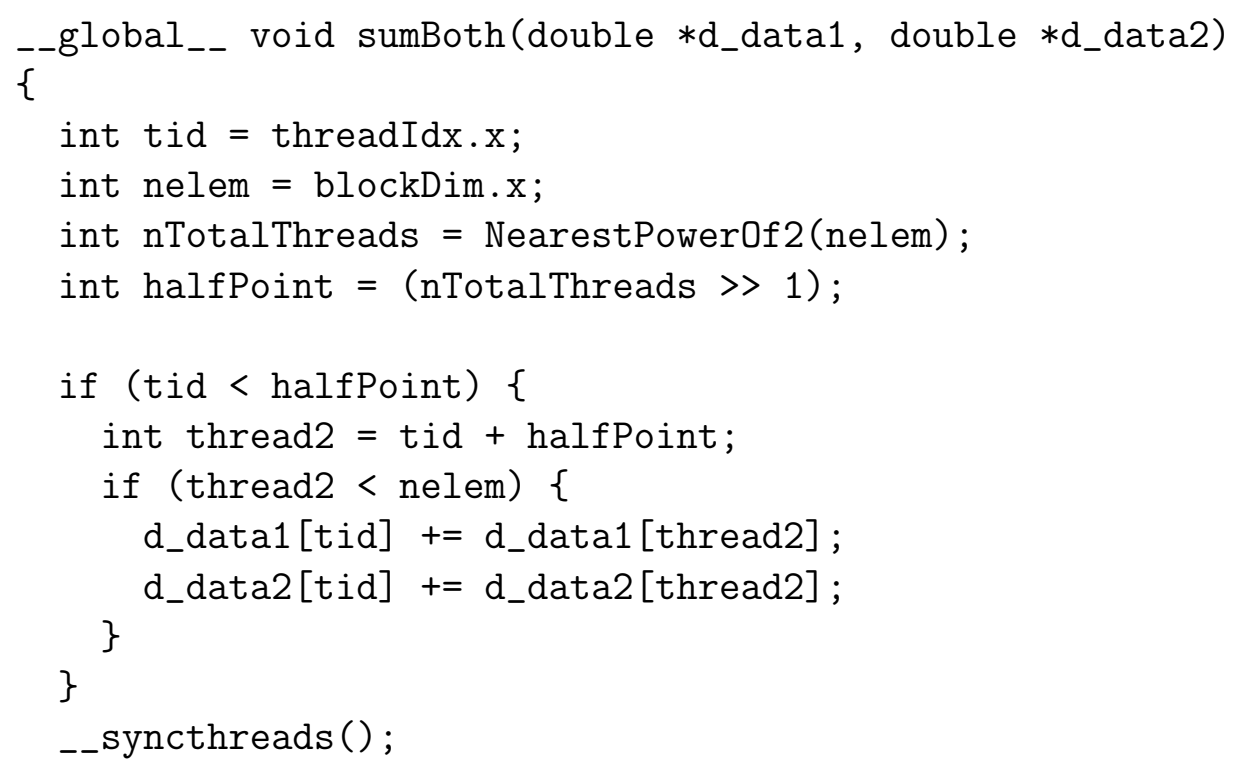




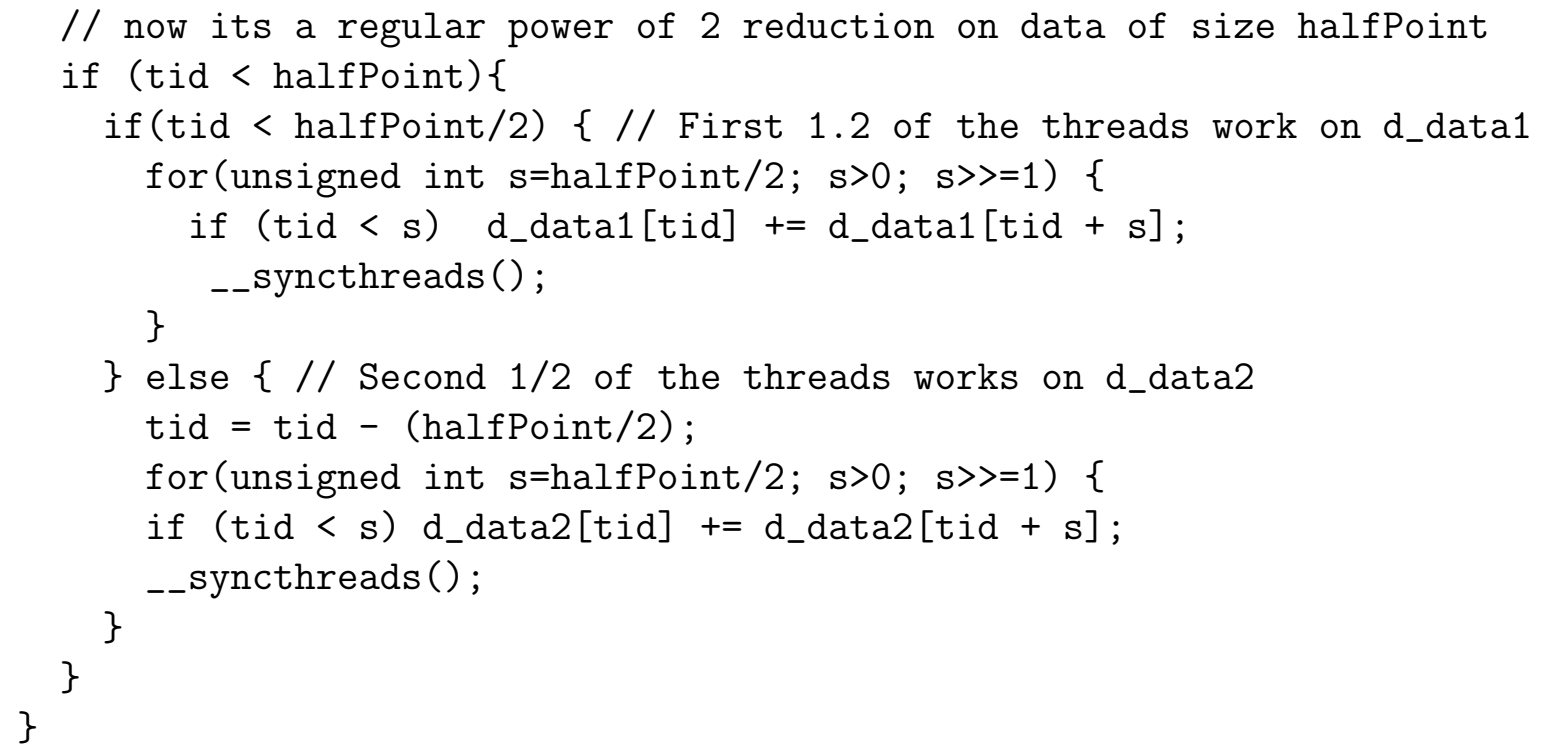

\section{References}

Barnett, S. (1979). Matrix Methods for Engineers and Scientists. McGraw-Hill.

Bayarri, M., Berger, J., Paulo, R., Sacks, J., Cafeo, J., Cavendish, J., Lin, C.-H., and Tu, J. (2007). "A Framework for Validation of Computer Models." Technometrics, 49, 2, $138-154$.

Cohn, D. A. (1996). "Neural Network Exploration using Optimal Experimental Design." In Advances in Neural Information Processing Systems, vol. 6(9), 679-686. Morgan Kaufmann Publishers.

Cressie, N. (1991). Statistics for Spatial Data, revised edition. John Wiley and Sons, Inc.

Cressie, N. and Johannesson, G. (2008). "Fixed Rank Kriging for Very Large Data Sets." Journal of the Royal Statistical Soceity, Series B, 70, 1, 209-226.

Eidsvik, J., Shaby, B. A., Reich, B. J., Wheeler, M., and Niemi, J. (2013). "Estimation and prediction in spatial models with block composite likelihoods." Journal of Computational and Graphical Statistics, 0, ja, null.

Franey, M., Ranjan, P., and Chipman, H. (2012). "A Short Note on Gaussian Process Modeling for Large Datasets using Graphics Processing Units." Tech. rep., Acadia University.

Furrer, R., Genton, M., and Nychka, D. (2006). "Covariance tapering for interpolation of large spatial datasets." Journal of Computational and Graphical Statistics, 15, 502-523. 
Gilboa, E., Saatçi, Y., and Cunningham, J. P. (2012). "Scaling Multidimensional Inference for Structured Gaussian Processes." ArXiv e-prints.

Gramacy, R. and Polson, N. (2011). "Particle Learning of Gaussian Process Models for Sequential Design and Optimization." Journal of Computational and Graphical Statistics, $20,1,102-118$.

Gramacy, R. B. (2013). laGP: Local approximate Gaussian process regression. R package version 1.0 .

Gramacy, R. B. and Apley, D. W. (2014). "Local Gaussian process approximation for large computer experiments." Journal of Computational and Graphical Statistics. to appear; see arXiv:1303.0383.

Gramacy, R. B. and Lee, H. K. H. (2009). "Adaptive Design and Analysis of Supercomputer Experiments." Technometrics, 51, 2, 130-145.

Haaland, B. and Qian, P. (2011). "Accurate Emulators for Large-Scale Computer Experiments." Annals of Statistics, 39, 6, 2974-3002.

Hagan, R. (2011). "Multi-GPU load Balancing for Simulation and Rendering." Ph.D. thesis, Virginia Polytechnic Institute and State University, Blackburgh, VA.

Jones, D., Schonlau, M., and Welch, W. J. (1998). "Efficient Global Optimization of Expensive Black Box Functions." Journal of Global Optimization, 13, 455-492.

Kaufman, C., Bingham, D., Habib, S., Heitmann, K., and Frieman, J. (2012). "Efficient Emulators of Computer Experiments Using Compactly Supported Correlation Functions, With An Application to Cosmology." Annals of Applied Statistics, 5, 4, 2470-2492.

Kennedy, M. and O'Hagan, A. (2001). "Bayesian Calibration of Computer Models (with discussion)." Journal of the Royal Statistical Society, Series B, 63, 425-464.

Kirk, D. B. and Wen-mei, W. H. (2010). Programming massively parallel processors: a hands-on approach. Morgan Kaufmann.

Lee, V. W., Kim, C., Chhugani, J., Deisher, M., Kim, D., Nguyen, A. D., Satish, N., Smelyanskiy, M., Chennupaty, S., Hammarlund, P., Singhal, R., and Dubey, P. (2010). "Debunking the 100X GPU vs. CPU myth: an evaluation of throughput computing on CPU and GPU." SIGARCH Comput. Archit. News, 38, 3, 451-460.

Morris, D., Mitchell, T., and Ylvisaker, D. (1993). "Bayesian Design and Analysis of Computer Experiments: Use of Derivatives in Surface Prediction." Technometrics, 35, 243-255.

Nychka, D., Wikle, C., and Royle, J. (2002). "Multiresolution Models for Nonstationary Spatial Covariance Functions." Statistical Modelling, 2, 315-331. 
Paciorek, C., Lipshitz, B., Zhuo, W., Prabhat, Kaufman, C., and Thomas, R. (2013). "Parallelizing Gaussian Process Calculations in R." Tech. rep., University of California, Berkeley. ArXiv:1303.0383.

Plumlee, M. (2013). "Efficient inference for random fields using sparse grid designs." Tech. rep., Georgia Tech. under review.

Pratola, M. T., Chipman, H., Gattiker, J., Higdon, D., McCulloch, R., and Rust, W. (2013). "Parallel Bayesian Additive Regression Trees." Journal of Computational and Graphical Statistics. To appear.

Quiñonero-Candela, J. and Rasmussen, C. (2005). "A Unifying View of Sparse Approximate Gaussian Process Regression." Journal of Machine Learning Research, 6, 1939-1959.

Ranjan, P., Haynes, R., and Karsten, R. (2011). "A Computationally Stable Approach to Gaussian Process Interpolation of Deterministic Computer Simulation Data." Technometrics, 53, 4, 363-378.

Rasmussen, C. E. and Williams, C. K. I. (2006). Gaussian Processes for Machine Learning. The MIT Press.

Sang, H. and Huang, J. Z. (2012). "A Full Scale Approximation of Covariance Functions for Large Spatial Data Sets." Journal of the Royal Statistical Society: Series B, 74, 1, $111-132$.

Santner, T. J., Williams, B. J., and Notz, W. I. (2003). The Design and Analysis of Computer Experiments. New York, NY: Springer-Verlag.

Seo, S., Wallat, M., Graepel, T., and Obermayer, K. (2000). "Gaussian Process Regression: Active Data Selection and Test Point Rejection." In Proceedings of the International Joint Conference on Neural Networks, vol. III, 241-246. IEEE.

SHARCNET (2012). CUDA Tips and Tricks.

Stein, M. L., Chi, Z., and Welty, L. J. (2004). "Approximating Likelihoods for Large Spatial Data Sets." Journal of the Royal Statistical Society, Series B, 66, 2, 275-296.

Tierney, L., Rossini, A. J., Li, N., and Sevcikova, H. (2013). snow: Simple Network of Workstations. R package version 0.3-12.

Vecchia, A. (1988). "Estimation and model identification for continuous spatial processes." Journal of the Royal Statistical Soceity, Series B, 50, 297-312.

Worley, B. (1987). "Deterministic Uncertainty Analysis." Tech. Rep. ORN-0628, National Technical Information Service, 5285 Port Royal Road, Springfield, VA 22161, USA. 